\title{
Global Analysis of Almost Periodic Solution of a Discrete Multispecies Mutualism System
}

\author{
Hui Zhang, Bin Jing, Yingqi Li, and Xiaofeng Fang \\ Mathematics and OR Section, Xi'an Research Institute of High-Tech, Hongqing Town, Xi'an, Shaanxi 710025, China \\ Correspondence should be addressed to Hui Zhang; zh53054958@163.com
}

Received 11 February 2014; Accepted 6 April 2014; Published 22 April 2014

Academic Editor: Yongkun Li

Copyright ( 2014 Hui Zhang et al. This is an open access article distributed under the Creative Commons Attribution License, which permits unrestricted use, distribution, and reproduction in any medium, provided the original work is properly cited.

\begin{abstract}
This paper discusses a discrete multispecies Lotka-Volterra mutualism system. We first obtain the permanence of the system. Assuming that the coefficients in the system are almost periodic sequences, we obtain the sufficient conditions for the existence of a unique almost periodic solution which is globally attractive. In particular, for the discrete two-species Lotka-Volterra mutualism system, the sufficient conditions for the existence of a unique uniformly asymptotically stable almost periodic solution are obtained. An example together with numerical simulation indicates the feasibility of the main result.
\end{abstract}

\section{Introduction}

In this paper, we consider a discrete multispecies LotkaVolterra mutualism system:

$$
\begin{array}{r}
x_{i}(k+1)=x_{i}(k) \exp \left\{a_{i}(k)-b_{i}(k) x_{i}(k)\right. \\
\left.+\sum_{j=1, j \neq i}^{n} c_{i j}(k) \frac{x_{j}(k)}{d_{i j}+x_{j}(k)}\right\}, \\
i=1,2, \ldots, n,
\end{array}
$$

where $x_{i}(k)$ stand for the densities of species $x_{i}$ at the $k$ th generation, $a_{i}(k)$ represent the natural growth rates of species $x_{i}$ at the $k$ th generation, $b_{i}(k)$ are the intraspecific effects of the $k$ th generation of species $x_{i}$ on own population, $c_{i j}(k)$ measure the interspecific mutualism effects of the $k$ th generation of species $x_{j}$ on species $x_{i}(i, j=1,2, \ldots, n, i \neq j)$, and $d_{i j}(\geq 1)$ are positive control constants.

A number of scholars have studied the difference system (see [1-8] and the references cited therein) since the discrete time models governed by the difference equation are more appropriate than the continuous ones when the populations have short life expectancy, nonoverlapping generations in the real world.

Recently, as far as the multispecies Lotka-Volterra ecosystem is concerned, Wendi and Zhengyi [1] proposed the following Lotka-Volterra model:

$$
\begin{array}{r}
x_{i}(k+1)=x_{i}(k) \exp \left[r_{i}(k)-\sum_{j=1}^{n} a_{i j}(k) x_{j}(k)\right], \\
i=1,2, \ldots, n .
\end{array}
$$

By constructing a suitable Lyapunov function and using the finite covering theorem of mathematic analysis, they obtained a set of sufficient conditions which ensure the system to be globally asymptotically stable.

Chen [3] studied the dynamic behavior of the discrete $n+$ $m$-species Lotka-Volterra competition predator-prey systems:

$$
\begin{gathered}
x_{i}(k+1)=x_{i}(k) \exp \left[b_{i}(k)-\sum_{l=1}^{n} a_{i l}(k) x_{l}(k)\right. \\
\left.-\sum_{l=1}^{m} c_{i l}(k) y_{l}(k)\right],
\end{gathered}
$$




$$
\begin{aligned}
y_{j}(k+1)=y_{j}(k) \exp [ & -r_{j}(k)+\sum_{l=1}^{n} d_{j l}(k) x_{l}(k) \\
& \left.\times \sum_{l=1}^{m} e_{j l}(k) y_{l}(k)\right]
\end{aligned}
$$

where $i=1,2, \ldots, n$ and $j=1,2, \ldots, m$. Sufficient conditions which ensure the permanence and the global stability of the systems are obtained; for periodic case, sufficient conditions which ensure the existence of a globally stable positive periodic solution of the systems are obtained.

At the same time, a few scholars have investigated the mutualism system (see $[2,8-13]$ in detail). However, to the best of the authors' knowledge, still no scholar has done works on discrete multispecies mutualism system. So we propose the discrete multispecies Lotka-Volterra mutualism system (1).

Notice that the investigation of almost periodic solutions for difference equations is one of the most important topics in the qualitative theory of difference equations due to the applications in biology, ecology, neural network, and so forth (see [7, 14-21] and the references cited therein), and little work has been done previously on an almost periodic version which is corresponding to system (1). Then, we will further investigate the existence of a unique almost periodic solution of system (1) which is globally attractive.

Denote as $Z$ and $Z^{+}$the set of integers and the set of nonnegative integers, respectively. For any bounded sequence $g(n)$ defined on $Z$, define $g^{u}=\sup _{n \in Z} g(n), g^{l}=\inf _{n \in Z} g(n)$.

Throughout this paper, we assume the following.

(H1) $a_{i}(k), b_{i}(k)$, and $c_{i j}(k)$ are bounded nonnegative almost periodic sequences such that

$$
\begin{gathered}
0<a_{i}^{l} \leq a_{i}(k) \leq a_{i}^{u}, \quad 0<b_{i}^{l} \leq b_{i}(k) \leq b_{i}^{u}, \\
0<c_{i j}^{l} \leq c_{i j}(k) \leq c_{i j}^{u} .
\end{gathered}
$$

From the point of view of biology, in the sequel, we assume that $\mathbf{x}(0)=\left(x_{1}(0), x_{2}(0), \ldots, x_{n}(0)\right)>\mathbf{0}$. Then, it is easy to see that, for given $\mathbf{x}(0)>\mathbf{0}$, the system (1) has a positive sequence solution $\mathbf{x}(k)=\left(x_{1}(k), x_{2}(k), \ldots, x_{n}(k)\right)(k \in$ $Z^{+}$) passing through $\mathbf{x}(0)$.

The remaining part of this paper is organized as follows. In Section 2, we will introduce some definitions and several useful lemmas. In Section 3, by applying the theory of difference inequality, we present the permanence results for system (1). In Section 4, we establish the sufficient conditions for the existence of a unique globally attractive almost periodic solution of system (1). In particular, for the discrete two-species Lotka-Volterra mutualism system, the sufficient conditions for the existence of a unique uniformly asymptotically stable almost periodic solution are obtained. The main result is illustrated by an example with a numerical simulation in the last section.

\section{Preliminaries}

Firstly, we give the definitions of the terminologies involved.

Definition 1 (see [22]). A sequence $x: Z \rightarrow R$ is called an almost periodic sequence if the $\varepsilon$-translation set of $x$

$$
E\{\varepsilon, x\}=\{\tau \in Z:|x(n+\tau)-x(n)|<\varepsilon, \forall n \in Z\}
$$

is a relatively dense set in $Z$ for all $\varepsilon>0$; that is, for any given $\varepsilon>0$, there exists an integer $l(\varepsilon)>0$ such that each interval of length $l(\varepsilon)$ contains an integer $\tau \in E\{\varepsilon, x\}$ with

$$
|x(n+\tau)-x(n)|<\varepsilon, \quad \forall n \in Z .
$$

$\tau$ is called an $\varepsilon$-translation number of $x(n)$.

Definition 2 (see [23]). A sequence $x: Z^{+} \rightarrow R$ is called an asymptotically almost periodic sequence if

$$
x(n)=p(n)+q(n), \quad \forall n \in Z^{+},
$$

where $p(n)$ is an almost periodic sequence and $\lim _{n \rightarrow \infty} q(n)$ $=0$.

Definition 3 (see [24]). A solution $\left(x_{1}(k), x_{2}(k), \ldots, x_{n}(k)\right)$ of system (1) is said to be globally attractive if, for any other solution $\left(x_{1}^{*}(k), x_{2}^{*}(k), \ldots, x_{n}^{*}(k)\right)$ of system (1), one has

$$
\lim _{k \rightarrow+\infty}\left(x_{i}^{*}(k)-x_{i}(k)\right)=0, \quad i=1,2, \ldots, n .
$$

Now, we present some results which will play an important role in the proof of the main result.

Lemma 4 (see [25]). If $\{x(n)\}$ is an almost periodic sequence, then $\{x(n)\}$ is bounded.

Lemma 5 (see [26]). $\{x(n)\}$ is an almost periodic sequence if and only if, for any sequence $m_{i} \subset Z$, there exists a subsequence $\left\{m_{i_{k}}\right\} \subset\left\{m_{i}\right\}$ such that the sequence $\left\{x\left(n+m_{i_{k}}\right)\right\}$ converges uniformly for all $n \in Z$ as $k \rightarrow \infty$. Furthermore, the limit sequence is also an almost periodic sequence.

Lemma 6 (see [23]). $\{x(n)\}$ is an asymptotically almost periodic sequence if and only if, for any sequence $m_{i} \subset Z$ satisfying $m_{i}>0$ and $m_{i} \rightarrow \infty$ as $i \rightarrow \infty$, there exists a subsequence $\left\{m_{i_{k}}\right\} \subset\left\{m_{i}\right\}$ such that the sequence $\left\{x\left(n+m_{i_{k}}\right)\right\}$ converges uniformly for all $n \in Z^{+}$as $k \rightarrow \infty$.

Lemma 7 (see [25]). Suppose that $\left\{p_{1}(n)\right\}$ and $\left\{p_{2}(n)\right\}$ are almost periodic real sequences. Then, $\left\{p_{1}(n)+p_{2}(n)\right\}$ and $\left\{p_{1}(n) p_{2}(n)\right\}$ are almost periodic; $1 / p_{1}(n)$ is also almost periodic provided that $p_{1}(n) \neq 0$ for all $n \in Z$. Moreover, if $\varepsilon>0$ is an arbitrary real number, then there exists a relatively dense set that is $\varepsilon$-almost periodic common to $\left\{p_{1}(n)\right\}$ and $\left\{p_{2}(n)\right\}$.

Lemma 8 (see $[3,11,27])$. Assume that $\{x(n)\}$ satisfies $x(n)>$ 0 and

$$
x(n+1) \leq x(n) \exp \{a(n)-b(n) x(n)\},
$$


for $n \in N$, where $a(n)$ and $b(n)$ are nonnegative sequences bounded above and below by positive constants. Then,

$$
\limsup _{n \rightarrow+\infty} x(n) \leq \frac{1}{b^{l}} \exp \left\{a^{u}-1\right\} .
$$

Lemma 9 (see $[3,11,27])$. Assume that $\{x(n)\}$ satisfies

$$
\begin{gathered}
x(n+1) \geq x(n) \exp \{a(n)-b(n) x(n)\}, \quad n \geq N_{0}, \\
\limsup _{n \rightarrow+\infty} x(n) \leq x^{*},
\end{gathered}
$$

and $x\left(N_{0}\right)>0$, where $a(n)$ and $b(n)$ are nonnegative sequences bounded above and below by positive constants and $N_{0} \in N$. Then,

$$
\liminf _{n \rightarrow+\infty} x(n) \geq \min \left\{\frac{a^{l}}{b^{u}} \exp \left\{a^{l}-b^{u} x^{*}\right\}, \frac{a^{l}}{b^{u}}\right\} .
$$

Consider the following almost periodic difference system:

$$
x(n+1)=f(n, x(n)), \quad n \in Z^{+},
$$

where $f: Z^{+} \times S_{B} \rightarrow R^{K}, S_{B}=\left\{x \in R^{k}:\|x\|<B\right\}$, and $f(n, x)$ is almost periodic in $n$ uniformly for $x \in S_{B}$ and is continuous in $x$. The product system of (13) is the following system:

$$
x(n+1)=f(n, x(n)), \quad y(n+1)=f(n, y(n)),
$$

and Zhang [28] obtained the following lemma.

Lemma 10 (see [28]). Suppose that there exists a Lyapunov function $V(n, x, y)$ defined for $n \in Z^{+},\|x\|<B$, and $\|y\|<B$ satisfying the following conditions:

(i) $a(\|x-y\|) \leq V(n, x, y) \leq b(\|x-y\|)$, where $a, b \in K$ with $K=\left\{a \in C\left(R^{+}, R^{+}\right): a(0)=\right.$ 0 and $a$ is increasing $\}$;

(ii) $\left\|V\left(n, x_{1}, y_{1}\right)-V\left(n, x_{2}, y_{2}\right)\right\| \leq L\left[\left\|x_{1}-x_{2}\right\|+\left\|y_{1}-y_{2}\right\|\right]$, where $L>0$ is a constant;

(iii) $\Delta V_{(14)}(n, x, y) \leq-\alpha V(n, x, y)$, where $0<\alpha<1$ is a constant, and

$\Delta V_{(14)}(n, x, y) \equiv V(n+1, f(n, x), f(n, y))-V(n, x, y)$.

Moreover, if there exists a solution $\varphi(n)$ of (13) such that $\|\varphi(n)\| \leq B^{*}<B$ for $n \in Z^{+}$, then there exists a unique uniformly asymptotically stable almost periodic solution $p(n)$ of (13) which is bounded by $B^{*}$. In particular, if $f(n, x)$ is periodic of period $\omega$, then there exists a unique uniformly asymptotically stable periodic solution of (13) of period $\omega$.

\section{Permanence}

In this section, we establish a permanence result for system (1), which can be found by Lemmas 8 and 9 .
Proposition 11. Assume that (H1) holds. Then, any positive solution $\left(x_{1}(k), x_{2}(k), \ldots, x_{n}(k)\right)$ of system (1) satisfies

$$
m_{i} \leq \liminf _{k \rightarrow+\infty} x_{i}(k) \leq \limsup _{k \rightarrow+\infty} x_{i}(k) \leq M_{i}, \quad i=1,2, \ldots, n,
$$

where

$$
\begin{gathered}
M_{i}=\frac{1}{b_{i}^{l}} \exp \left\{a_{i}^{u}+\sum_{j=1, j \neq i}^{n} c_{i j}^{u}-1\right\}, \\
m_{i}=\min \left\{\frac{a_{i}^{l}}{b_{i}^{u}} \exp \left(a_{i}^{l}-b_{i}^{u} M_{i}\right), \frac{a_{i}^{l}}{b_{i}^{u}}\right\} .
\end{gathered}
$$

Theorem 12. Assume that (H1) holds; then system (1) is permanent.

It should be noticed that, from Proposition 11, we know that the set

$$
\left[m_{1}, M_{1}\right] \times\left[m_{2}, M_{2}\right] \times \cdots \times\left[m_{n}, M_{n}\right]
$$

is an invariant set of system (1).

The next result tells us that there exist solutions of system (1) totally in the interval of Proposition 11. To be precise, see the following.

Proposition 13. System (1) has a solution $\left(x_{1}(k), x_{2}(k)\right.$, $\left.\ldots, x_{n}(k)\right)$ satisfying $m_{i} \leq x_{i}(k) \leq M_{i}$ for $k \in Z$.

Proof. By the almost periodicity of $\left\{a_{i}(k)\right\},\left\{b_{i}(k)\right\}$, and $\left\{c_{i j}(k)\right\}$, there exists an integer valued sequence $\left\{\delta_{p}\right\}$ with $\delta_{p} \rightarrow \infty$ as $p \rightarrow \infty$ such that

$$
\begin{array}{r}
a_{i}\left(k+\delta_{p}\right) \longrightarrow a_{i}(k), \\
b_{i}\left(k+\delta_{p}\right) \longrightarrow b_{i}(k), \\
c_{i j}\left(k+\delta_{p}\right) \longrightarrow c_{i j}(k) \\
\text { as } p \longrightarrow+\infty .
\end{array}
$$

Let $\varepsilon$ be an arbitrary small positive number. It follows from Proposition 11 that there exists a positive integer $N_{0}$ such that

$$
m_{i}-\varepsilon \leq x_{i}(k) \leq M_{i}+\varepsilon, \quad k>N_{0} .
$$

Write $x_{i p}(k)=x_{i}\left(k+\delta_{p}\right)$ for $k \geq N_{0}-\delta_{p}$ and $p=1,2, \ldots$ For any positive integer $q$, it is easy to see that there exists a sequence $\left\{x_{i p}(k): p \geq q\right\}$ such that the sequence $x_{p}(k)$ has a subsequence, denoted by $\left\{x_{i p}(k)\right\}$ again, converging on any finite interval of $Z$ as $p \rightarrow \infty$. Thus, we have a sequence $\left\{y_{i}(k)\right\}$ such that

$$
x_{i p}(k) \longrightarrow y_{i}(k) \quad \text { for } k \in Z \text { as } p \longrightarrow \infty \text {. }
$$


This, combined with

$$
\begin{aligned}
x_{i}\left(k+1+\delta_{p}\right) & \\
= & x_{i}\left(k+\delta_{p}\right) \\
\times & \quad\left\{\operatorname { e x p } \left\{a_{i}\left(k+\delta_{p}\right)\right.\right. \\
& -b_{i}\left(k+\delta_{p}\right) x_{i}\left(k+\delta_{p}\right) \\
& \left.+\sum_{j=1, j \neq i}^{n} c_{i j}\left(k+\delta_{p}\right) \frac{x_{j}\left(k+\delta_{p}\right)}{d_{i j}+x_{j}\left(k+\delta_{p}\right)}\right\}, \\
& i=1,2, \ldots, n,
\end{aligned}
$$

gives us

$$
\begin{array}{r}
y_{i}(k+1)=y_{i}(k) \exp \left\{a_{i}(k)-b_{i}(k) y_{i}(k)\right. \\
\left.+\sum_{j=1, j \neq i}^{n} c_{i j}(k) \frac{y_{j}(k)}{d_{i j}+y_{j}(k)}\right\}, \\
i=1,2, \ldots, n .
\end{array}
$$

We can easily see that $\left\{y_{i}(k)\right\}$ is a solution of system (1) and $m_{i}-\varepsilon \leq y_{i}(k) \leq M_{i}+\varepsilon$ for $k \in Z$. Since $\varepsilon$ is an arbitrary small positive number, it follows that $m_{i} \leq y_{i}(k) \leq M_{i}$, and hence we complete the proof.

\section{Main Result}

The main result of this paper concerns the existence of a globally attractive almost periodic solution of system (1).

Theorem 14. Assume that (H1) and

(H2)

$$
\begin{aligned}
\rho_{i}= & \max \left\{\left|1-b_{i}^{l} m_{i}\right|,\left|1-b_{i}^{u} M_{i}\right|\right\} \\
& +\sum_{j=1, j \neq i}^{n} c_{i j}^{u} M_{j}<1, \quad i=1,2, \ldots, n,
\end{aligned}
$$

hold. Then, system (1) admits a unique almost periodic solution which is globally attractive.

Proof. It follows from Proposition 13 that there exists a solution $\left(x_{1}(k), x_{2}(k), \ldots, x_{n}(k)\right)$ of system (1) satisfying $m_{i} \leq$ $x_{i}(k) \leq M_{i}, k \in Z^{+}$. Let $\left\{\delta_{k}\right\}$ be any integer valued sequence such that $\delta_{k} \rightarrow \infty$ as $k \rightarrow \infty$. Using the mean value theorem, for $p \neq q$, we get

$$
\begin{aligned}
\ln x_{i} & \left(k+\delta_{p}\right)-\ln x_{i}\left(k+\delta_{q}\right) \\
= & \frac{1}{\xi_{i}(k, p, q)}\left[x_{i}\left(k+\delta_{p}\right)-x_{i}\left(k+\delta_{q}\right)\right],
\end{aligned}
$$

where $\xi_{i}(k, p, q)$ lies between $x_{i}\left(k+\delta_{p}\right)$ and $x_{i}\left(k+\delta_{q}\right)$. Then,

$$
\begin{aligned}
& \quad\left|x_{i}\left(k+\delta_{p}\right)-x_{i}\left(k+\delta_{q}\right)\right| \\
& \quad \leq M_{i}\left|\ln x_{i}\left(k+\delta_{p}\right)-\ln x_{i}\left(k+\delta_{q}\right)\right|, \quad k \in Z^{+} .
\end{aligned}
$$

For convenience, we introduce $\varphi_{i}\left(k, \delta_{p}, \delta_{q}\right)$ through

$$
\begin{array}{r}
\varphi_{i}\left(k, \delta_{p}, \delta_{q}\right)=\left|\ln x_{i}\left(k+\delta_{p}\right)-\ln x_{i}\left(k+\delta_{q}\right)\right|, \\
k \in Z^{+}, \quad \delta_{p}>0, \quad \delta_{q}>0 .
\end{array}
$$

Thus,

$$
\begin{aligned}
& \varphi_{i}\left(k+1, \delta_{p}, \delta_{q}\right) \\
& =\left|\ln x_{i}\left(k+1+\delta_{p}\right)-\ln x_{i}\left(k+1+\delta_{q}\right)\right| \\
& =\mid \ln x_{i}\left(k+\delta_{p}\right)-\ln x_{i}\left(k+\delta_{q}\right)+a_{i}\left(k+\delta_{p}\right) \\
& -a_{i}\left(k+\delta_{q}\right)-b_{i}\left(k+\delta_{p}\right) x_{i}\left(k+\delta_{p}\right) \\
& +b_{i}\left(k+\delta_{q}\right) x_{i}\left(k+\delta_{q}\right) \\
& +\sum_{j=1, j \neq i}^{n} c_{i j}\left(k+\delta_{p}\right) \frac{x_{j}\left(k+\delta_{p}\right)}{d_{i j}+x_{j}\left(k+\delta_{p}\right)} \\
& -\sum_{j=1, j \neq i}^{n} c_{i j}\left(k+\delta_{q}\right) \frac{x_{j}\left(k+\delta_{q}\right)}{d_{i j}+x_{j}\left(k+\delta_{q}\right)} \mid \\
& \leq \mid \ln x_{i}\left(k+\delta_{p}\right)-\ln x_{i}\left(k+\delta_{q}\right)-b_{i}\left(k+\delta_{p}\right) \\
& \times\left[x_{i}\left(k+\delta_{p}\right)-x_{i}\left(k+\delta_{q}\right)\right] \mid \\
& +\left|a_{i}\left(k+\delta_{p}\right)-a_{i}\left(k+\delta_{q}\right)\right| \\
& +\left|\left[b_{i}\left(k+\delta_{q}\right)-b_{i}\left(k+\delta_{p}\right)\right] x_{i}\left(k+\delta_{q}\right)\right| \\
& +\sum_{j=1, j \neq i}^{n} \mid c_{i j}\left(k+\delta_{p}\right)\left[\frac{x_{j}\left(k+\delta_{p}\right)}{d_{i j}+x_{j}\left(k+\delta_{p}\right)}\right. \\
& \left.-\frac{x_{j}\left(k+\delta_{q}\right)}{d_{i j}+x_{j}\left(k+\delta_{q}\right)}\right] \\
& +\sum_{j=1, j \neq i}^{n} \mid\left[c_{i j}\left(k+\delta_{p}\right)-c_{i j}\left(k+\delta_{q}\right)\right] \\
& \times \frac{x_{j}\left(k+\delta_{q}\right)}{d_{i j}+x_{j}\left(k+\delta_{q}\right)} \mid \\
& \leq \mid \ln x_{i}\left(k+\delta_{p}\right)-\ln x_{i}\left(k+\delta_{q}\right)-b_{i}\left(k+\delta_{p}\right) \\
& \times\left[x_{i}\left(k+\delta_{p}\right)-x_{i}\left(k+\delta_{q}\right)\right] \mid \\
& +\left|a_{i}\left(k+\delta_{p}\right)-a_{i}\left(k+\delta_{q}\right)\right|
\end{aligned}
$$




$$
\begin{aligned}
& +\left|\left[b_{i}\left(k+\delta_{q}\right)-b_{i}\left(k+\delta_{p}\right)\right] x_{i}\left(k+\delta_{q}\right)\right| \\
& +\sum_{j=1, j \neq i}^{n}\left|c_{i j}\left(k+\delta_{p}\right)\left[x_{j}\left(k+\delta_{p}\right)-x_{j}\left(k+\delta_{q}\right)\right]\right| \\
& +\sum_{j=1, j \neq i}^{n}\left|\left[c_{i j}\left(k+\delta_{p}\right)-c_{i j}\left(k+\delta_{q}\right)\right] x_{j}\left(k+\delta_{q}\right)\right| .
\end{aligned}
$$

Let $\varepsilon_{1}$ be an arbitrary positive number. By the almost periodicity of $\left\{a_{i}(k)\right\},\left\{b_{i}(k)\right\}$, and $\left\{c_{i j}(k)\right\}$ and the boundedness of $\left\{\left(x_{1}(k), x_{2}(k), \ldots, x_{n}(k)\right)\right\}$, it follows from Lemmas 5 and 7 that there exists a positive integer $K_{1}=K_{1}\left(\varepsilon_{1}\right)$ such that, for any $\delta_{q} \geq \delta_{p} \geq K_{1}$ and $k \in Z^{+}$(if necessary, we can choose subsequences of $\left\{\delta_{p}\right\}$ and $\left\{\delta_{q}\right\}$ ),

$$
\begin{gathered}
\left|a_{i}\left(k+\delta_{p}\right)-a_{i}\left(k+\delta_{q}\right)\right|<\frac{\varepsilon_{1}}{3}, \\
\left|\left[b_{i}\left(k+\delta_{q}\right)-b_{i}\left(k+\delta_{p}\right)\right] x_{i}\left(k+\delta_{q}\right)\right|<\frac{\varepsilon_{1}}{3}, \\
\sum_{j=1, j \neq i}^{n}\left|\left[c_{i j}\left(k+\delta_{p}\right)-c_{i j}\left(k+\delta_{q}\right)\right] x_{j}\left(k+\delta_{q}\right)\right| \\
<\frac{\varepsilon_{1}}{3} .
\end{gathered}
$$

It follows from (25) and (27)-(29) that, for $k \in Z^{+}$and $\delta_{q} \geq$ $\delta_{q} \geq K_{1}$,

$$
\begin{aligned}
\varphi_{i}\left(k+1, \delta_{p}, \delta_{q}\right)< & \left|1-b_{i}\left(k+\delta_{p}\right) \xi_{i}\left(k, \delta_{p}, \delta_{q}\right)\right| \\
& \times \varphi_{i}\left(k, \delta_{p}, \delta_{q}\right) \\
& +\sum_{j=1, j \neq i}^{n}\left|c_{i j}\left(k+\delta_{p}\right) \xi_{j}\left(k, \delta_{p}, \delta_{q}\right)\right| \\
& \times \varphi_{j}\left(k, \delta_{p}, \delta_{q}\right)+\varepsilon_{1} \\
\leq & \rho_{i} \max \left\{\varphi_{i}\left(k, \delta_{p}, \delta_{q}\right)\right\}+\varepsilon_{1} .
\end{aligned}
$$

Then,

$$
\begin{aligned}
\varphi_{i}\left(k, \delta_{p}, \delta_{q}\right) & <\rho_{i} \max \left\{\varphi_{i}\left(k-1, \delta_{p}, \delta_{q}\right)\right\}+\varepsilon_{1}, \\
\varphi_{i}\left(k-1, \delta_{p}, \delta_{q}\right) & <\rho_{i} \max \left\{\varphi_{i}\left(k-2, \delta_{p}, \delta_{q}\right)\right\}+\varepsilon_{1}, \\
& \vdots \\
\varphi_{i}\left(1, \delta_{p}, \delta_{q}\right) & <\rho_{i} \max \left\{\varphi_{i}\left(0, \delta_{p}, \delta_{q}\right)\right\}+\varepsilon_{1} .
\end{aligned}
$$

And we have

$$
\varphi_{i}\left(k, \delta_{p}, \delta_{q}\right)<\rho_{i}^{k} \max \left\{\varphi_{i}\left(0, \delta_{p}, \delta_{q}\right)\right\}+\frac{1-\rho_{i}^{k}}{1-\rho_{i}} \varepsilon_{1},
$$

for $k \in Z^{+}$and $\delta_{q} \geq \delta_{q} \geq K_{1}$.
Since $\rho_{i}<1$, for arbitrary $\varepsilon>0$, there exists a positive integer $K=K(\varepsilon)>K_{1}$ such that, for any $\delta_{q} \geq \delta_{p} \geq K$,

$$
\varphi_{i}\left(k, \delta_{p}, \delta_{q}\right)<\frac{\varepsilon}{\max _{1 \leq i \leq n}\left\{M_{i}\right\}}
$$

for $k \in Z^{+}$.

This combined with (26) gives us

$$
\begin{array}{r}
\left|x_{i}\left(k+\delta_{p}\right)-x_{i}\left(k+\delta_{q}\right)\right|<\varepsilon \\
\quad \text { for } k \in Z^{+}, \quad \delta_{q} \geq \delta_{q} \geq K .
\end{array}
$$

It follows from Lemma 6 that the sequence $\left\{x_{i}(k)\right\}(i=$ $1,2, \ldots, n)$ is asymptotically almost periodic. Thus, we can express $\left\{x_{i}(k)\right\}$ as

$$
x_{i}(k)=p_{i}(k)+q_{i}(k)
$$

where $\left\{p_{i}(k)\right\}$ are almost periodic in $k \in Z$ and $q_{i}(k) \rightarrow 0$ as $k \rightarrow \infty$. In the following, we show that $\left\{p_{i}(k)\right\}(i=$ $1,2, \ldots, n)$ is an almost periodic solution of system (1).

Define

$$
\begin{aligned}
f_{i}(k)= & a_{i}(k)-b_{i}(k)\left[p_{i}(k)+q_{i}(k)\right] \\
& +\sum_{j=1, j \neq i}^{n} c_{i j}(k) \frac{p_{j}(k)+q_{j}(k)}{d_{i j}+p_{j}(k)+q_{j}(k)}, \\
g_{i}(k)= & a_{i}(k)-b_{i}(k) p_{i}(k) \\
& +\sum_{j=1, j \neq i}^{n} c_{i j}(k) \frac{p_{j}(k)}{d_{i j}+p_{j}(k)}, \quad i=1,2, \ldots, n .
\end{aligned}
$$

It follows from (1), (35), and the mean value theorem that

$$
\begin{aligned}
& p_{i}(k+1)+q_{i}(k+1) \\
& =\left[p_{i}(k)+q_{i}(k)\right] \exp \left[f_{i}(k)\right] \\
& =p_{i}(k)\left\{\exp \left[f_{i}(k)\right]-\exp \left[g_{i}(k)\right]\right\} \\
& +p_{i}(k) \exp \left[g_{i}(k)\right]+q_{i}(k) \exp \left[f_{i}(k)\right] \\
& =-p_{i}(k) \exp \left[\xi_{i}(k)\right] \\
& \times\left[b_{i}(k) q_{i}(k)\right. \\
& +\sum_{j=1, j \neq i}^{n} c_{i j}(k)\left(\frac{p_{j}(k)}{d_{i j}+p_{j}(k)}\right. \\
& \left.\left.-\frac{p_{j}(k)+q_{j}(k)}{d_{i j}+p_{j}(k)+q_{j}(k)}\right)\right] \\
& +p_{i}(k) \exp \left[g_{i}(k)\right]+q_{i}(k) \exp \left[f_{i}(k)\right],
\end{aligned}
$$


where $\xi_{i}(k)=\theta_{i}(k) f_{i}(k)+\left(1-\theta_{i}(k)\right) g_{i}(k)$ for some $\theta_{i}(k) \epsilon$ $[0,1]$. Thus,

$$
\begin{aligned}
p_{i}(k+1) & -p_{i}(k) \exp \left[g_{i}(k)\right] \\
= & -p_{i}(k) \exp \left[\xi_{i}(k)\right] \\
& \times\left[b_{i}(k) q_{i}(k)\right. \\
& \left.\quad-\sum_{j=1, j \neq i}^{n} \frac{d_{i j} c_{i j}(k) q_{j}(k)}{\left(d_{i j}+p_{j}(k)\right)\left(d_{i j}+p_{j}(k)+q_{j}(k)\right)}\right] \\
- & q_{i}(k+1)+q_{i}(k) \exp \left[f_{i}(k)\right] .
\end{aligned}
$$

Let

$$
V_{i}(k)=p_{i}(k+1)-p_{i}(k) \exp \left[g_{i}(k)\right] .
$$

By the boundedness of the almost periodic sequences $\left\{a_{i}(k)\right\}$, $\left\{b_{i}(k)\right\},\left\{c_{i j}(k)\right\}$, and $\left\{p_{i}(k)\right\}$ and the fact that $q_{i}(k) \rightarrow 0$ as $k \rightarrow \infty$, we obtain

$$
V_{i}(k) \longrightarrow 0 \text { as } k \longrightarrow \infty
$$

We claim that $V_{i}(k) \equiv 0$. Otherwise, there exists an integer $k_{0} \in Z$ such that $V_{i}\left(k_{0}\right) \neq 0$. By the almost periodicity of $\left\{a_{i}(k)\right\},\left\{b_{i}(k)\right\},\left\{c_{i j}(k)\right\}$, and $\left\{p_{i}(k)\right\}$, there exists an integer valued sequence $\tau_{p}$ such that $\tau_{p} \rightarrow \infty$ as $p \rightarrow \infty$ and

$$
\begin{array}{ll}
a_{i}\left(k+\tau_{p}\right) \longrightarrow a_{i}(k), & b_{i}\left(k+\tau_{p}\right) \longrightarrow b_{i}(k), \\
c_{i j}\left(k+\tau_{p}\right) \longrightarrow c_{i j}(k), & p_{i}\left(k+\tau_{p}\right) \longrightarrow p_{i}(k)
\end{array}
$$

uniformly for all $k \in Z$. Then, we have

$$
\begin{aligned}
V_{i}\left(k_{0}+\tau_{p}\right)= & p_{i}\left(k_{0}+\tau_{p}+1\right) \\
& -p_{i}\left(k_{0}+\tau_{p}\right) \exp \left[g_{i}\left(k_{0}+\tau_{p}\right)\right] \\
& \longrightarrow p_{i}\left(k_{0}+1\right)-p_{i}\left(k_{0}\right) \exp \left[g_{i}\left(k_{0}\right)\right] \\
= & V_{i}\left(k_{0}\right)
\end{aligned}
$$

as $p \rightarrow \infty$, which contradicts the fact that $V_{i}(k) \rightarrow 0$ as $k \rightarrow \infty$. This proves the claim. Hence,

$$
p_{i}(k+1)=p_{i}(k) \exp \left[g_{i}(k)\right] ;
$$

that is, $\left\{p_{i}(k)\right\}$ is an almost periodic solution of system (1).

Assume that $\left(x_{1}(k), x_{2}(k), \ldots, x_{n}(k)\right)$ is the solution of system (1) satisfying (H1). Let

$$
x_{i}(k)=p_{i}(k) \exp \left(u_{i}(k)\right), \quad i=1,2, \ldots, n .
$$

Then, system (1) is equivalent to

$$
\begin{array}{r}
u_{i}(k+1) \\
=u_{i}(k)-b_{i}(k) p_{i}(k)\left[\exp \left(u_{i}(k)\right)-1\right] \\
+\sum_{j=1, j \neq i}^{n} c_{i j}(k) \frac{d_{i j} p_{j}(k)\left[\exp \left(u_{j}(k)\right)-1\right]}{\left[d_{i j}+p_{j}(k) \exp \left(u_{j}(k)\right)\right]\left[d_{i j}+p_{j}(k)\right]}, \\
i=1,2, \ldots, n .
\end{array}
$$

Therefore,

$$
\begin{array}{r}
u_{i}(k+1) \\
=u_{i}(k)\left[1-b_{i}(k) p_{i}(k) \exp \left(\theta_{i}(k) u_{i}(k)\right)\right] \\
+\sum_{j=1, j \neq i}^{n} c_{i j}(k) \frac{d_{i j} u_{j}(k) p_{j}(k) \exp \left(\overline{\theta_{j}}(k) u_{j}(k)\right)}{\left[d_{i j}+p_{j}(k) \exp \left(u_{j}(k)\right)\right]\left[d_{i j}+p_{j}(k)\right]}, \\
i=1,2, \ldots, n,
\end{array}
$$

where $\theta_{i}(k), \overline{\theta_{j}}(k) \in[0,1]$. To complete the proof, it suffices to show that

$$
\lim _{k \rightarrow \infty} u_{i}(k)=0, \quad i=1,2, \ldots, n .
$$

In view of (H2), we can choose $\varepsilon>0$ such that

$$
\begin{aligned}
\rho_{i}^{\varepsilon}= & \max \left\{\left|1-b_{i}^{l}\left(m_{i}-\varepsilon\right)\right|,\left|1-b_{i}^{u}\left(M_{i}+\varepsilon\right)\right|\right\} \\
& +\sum_{j=1, j \neq i}^{n} c_{i j}^{u}\left(M_{j}+\varepsilon\right)<1, \quad i=1,2, \ldots, n .
\end{aligned}
$$

Let $\rho=\max \left\{\rho_{i}^{\varepsilon}\right\}$; then $\rho<1$. According to Proposition 11, there exists a positive integer $k_{0} \in Z^{+}$such that

$$
\begin{array}{r}
m_{i}-\varepsilon \leq x_{i}(k) \leq M_{i}+\varepsilon, \\
m_{i}-\varepsilon \leq p_{i}(k) \leq M_{i}+\varepsilon, \\
i=1,2, \ldots, n,
\end{array}
$$

for $k \geq k_{0}$.

Notice that $\theta_{i}(k) \in[0,1]$ implies that $p_{i}(k) \exp \left(\theta_{i}(k) u_{i}\right.$ $(k)$ ) lies between $p_{i}(k)$ and $x_{i}(k) ; \overline{\theta_{j}}(k) \in[0,1]$ implies that $p_{j}(k) \exp \left(\overline{\theta_{j}}(k) u_{j}(k)\right)$ lies between $p_{j}(k)$ and $x_{j}(k)$. From (46), we get

$$
\begin{array}{r}
\left|u_{i}(k+1)\right| \leq \max \left\{\left|1-b_{i}^{l}\left(m_{i}-\varepsilon\right)\right|,\left|1-b_{i}^{u}\left(M_{i}+\varepsilon\right)\right|\right\} \\
\times\left|u_{i}(k)\right|+\sum_{j=1, j \neq i}^{n} c_{i j}^{u}\left(M_{j}^{u}+\varepsilon\right)\left|u_{j}(k)\right|, \\
i=1,2, \ldots, n,
\end{array}
$$

for $k \geq k_{0}$. 
In view of (50), we get

$$
\max _{1 \leq i \leq n}\left|u_{i}(k+1)\right| \leq \rho \max _{1 \leq i \leq n}\left|u_{i}(k)\right|, \quad k \geq k_{0} .
$$

This implies

$$
\max _{1 \leq i \leq n}\left|u_{i}(k)\right| \leq \rho^{k-k_{0}} \max _{1 \leq i \leq n}\left|u_{i}\left(k_{0}\right)\right|, \quad k \geq k_{0} .
$$

Then, (47) holds, and we can obtain

$$
\lim _{k \rightarrow+\infty}\left|x_{i}(k)-p_{i}(k)\right|=0, \quad i=1,2, \ldots, n .
$$

Therefore, system (1) admits a unique almost periodic solution which is globally attractive. This ends the proof of Theorem 14.

In particular, if $n=2$, we can obtain a discrete two-species Lotka-Volterra mutualism system:

$$
\begin{array}{r}
x_{1}(n+1)=x_{1}(n) \exp \left\{a_{1}(n)-b_{1}(n) x_{1}(n)\right. \\
\left.+c_{12}(n) \frac{x_{2}(n)}{d_{12}+x_{2}(n)}\right\}, \\
x_{2}(n+1)=x_{2}(n) \exp \left\{a_{2}(n)-b_{2}(n) x_{2}(n)\right. \\
\left.+c_{21}(n) \frac{x_{1}(n)}{d_{21}+x_{1}(n)}\right\} .
\end{array}
$$

In the following, the main result concerns the existence of a uniformly asymptomatically stable almost periodic solution of system (54).

From Proposition 11, we denote by $\Omega$ the set of all solutions $\left(x_{1}(n), x_{2}(n)\right)$ of system (54) satisfying $m_{i} \leq x_{i}(n) \leq$ $M_{i}, i=1,2$, for all $n \in Z^{+}$. According to Lemma 10, we first prove that there is a bounded solution of system (54) and then structure a suitable Lyapunov function for system (54).

Proposition 15. Assume that (H1) holds. Then, $\Omega \neq \Phi$.

Proof. By an inductive argument, we have from system (54) that

$$
\begin{array}{r}
x_{1}(n)=x_{1}(0) \exp \sum_{l=0}^{n-1}\left[a_{1}(l)-b_{1}(l) x_{1}(l)\right. \\
\left.+c_{12}(l) \frac{x_{2}(l)}{d_{12}+x_{2}(l)}\right], \\
x_{2}(n)=x_{2}(0) \exp \sum_{l=0}^{n-1}\left[a_{2}(l)-b_{2}(l) x_{1}(l)\right. \\
\left.+c_{21}(l) \frac{x_{1}(l)}{d_{21}+x_{1}(l)}\right] .
\end{array}
$$

According to Proposition 11, for any solution $\left(x_{1}(n), x_{2}(n)\right)$ of system (54) and an arbitrarily small constant $\varepsilon>0$, there exists $n_{0}$ sufficiently large such that

$$
\begin{array}{r}
m_{1}-\varepsilon \leq x_{1}(n) \leq M_{1}+\varepsilon, \\
m_{2}-\varepsilon \leq x_{2}(n) \leq M_{2}+\varepsilon, \\
\forall n \geq n_{0} .
\end{array}
$$

Set $\left\{\tau_{k}\right\}$ to be any positive integer sequence such that $\tau_{k} \rightarrow$ $+\infty$ as $k \rightarrow+\infty$; we can show that there exists a subsequence of $\left\{\tau_{k}\right\}$ still denoted by $\left\{\tau_{k}\right\}$, such that $x_{i}\left(k+\tau_{k}\right) \rightarrow x_{i}^{*}(k), i=$ 1,2 , uniformly in $n$ on any finite subset $C$ of $Z^{+}$as $k \rightarrow+\infty$, where $C=\left\{a_{1}, a_{2}, \ldots, a_{m}\right\}, a_{h} \in Z^{+}(h=1,2, \ldots, m)$, and $m$ is a finite number.

As a matter of fact, for any finite subset $C \subset Z^{+}, \tau_{k}+$ $a_{h}>n_{0}, h=1,2, \ldots, m$, when $k$ is large enough. Therefore, $m_{i}-\varepsilon \leq x_{i}\left(n+\tau_{k}\right) \leq M_{i}+\varepsilon, i=1,2$; that is, $x_{i}\left(n+\tau_{k}\right)$ are uniformly bounded for $k$ large enough.

Now, for $a_{1} \in C$, we can choose a subsequence $\left\{\tau_{k}^{(1)}\right\}$ of $\left\{\tau_{k}\right\}$ such that $\left\{x_{1}\left(a_{1}+\tau_{k}^{(1)}\right)\right\}$ and $\left\{x_{2}\left(a_{1}+\tau_{k}^{(1)}\right)\right\}$ uniformly converge on $Z^{+}$for $k$ large enough.

Analogously, for $a_{2} \in C$, we can also choose a subsequence $\left\{\tau_{k}^{(2)}\right\}$ of $\left\{\tau_{k}^{(1)}\right\}$ such that $\left\{x_{1}\left(a_{2}+\tau_{k}^{(2)}\right)\right\}$ and $\left\{x_{2}\left(a_{2}+\right.\right.$ $\left.\left.\tau_{k}^{(2)}\right)\right\}$ uniformly converge on $Z^{+}$for $k$ large enough.

Repeating the above process, for $a_{m} \in C$, we get a subsequence $\left\{\tau_{k}^{(m)}\right\}$ of $\left\{\tau_{k}^{(m-1)}\right\}$ such that $\left\{x_{1}\left(a_{m}+\tau_{k}^{(m)}\right)\right\}$ and $\left\{x_{2}\left(a_{m}+\tau_{k}^{(m)}\right)\right\}$ uniformly converge on $Z^{+}$for $k$ large enough.

Now, we choose the sequence $\left\{\tau_{k}^{(m)}\right\}$ which is a subsequence of $\left\{\tau_{k}\right\}$ denoted by $\left\{\tau_{k}\right\}$; then, for all $n \in C$, we obtain that $x_{i}\left(n+\tau_{k}\right) \rightarrow x_{i}^{*}(n), i=1,2$, uniformly in $n \in C$ as $k \rightarrow+\infty$. Hence, the conclusion is valid by the arbitrariness of $C$.

Recall the almost periodicity of $\left\{a_{i}(n)\right\},\left\{b_{i}(n)\right\}$, and $\left\{c_{i j}(n)\right\}$, for the above sequence $\left\{\tau_{k}\right\}, \tau_{k} \rightarrow+\infty$ as $k \rightarrow+\infty$; there exists a subsequence denoted by $\left\{\tau_{k}\right\}$ such that

$$
\begin{array}{r}
a_{i}\left(n+\tau_{k}\right) \longrightarrow a_{i}(n), \\
b_{i}\left(n+\tau_{k}\right) \longrightarrow b_{i}(n), \\
c_{i j}\left(n+\tau_{k}\right) \longrightarrow c_{i j}(n), \\
\quad i, j=1,2, \quad i \neq j,
\end{array}
$$

as $k \rightarrow+\infty$ uniformly on $Z^{+}$.

For any $\alpha \in Z^{+}$, we can assume that $\tau_{k}+\alpha \geq n_{0}$ for $k$ large enough. Let $n \in Z^{+}$, and, by an inductive argument of system (54) from $\tau_{k}+\alpha$ to $n+\tau_{k}+\alpha$, we obtain

$$
\begin{aligned}
& x_{1}\left(n+\tau_{k}+\alpha\right) \\
&=x_{1}\left(\tau_{k}+\alpha\right) \exp \sum_{l=\tau_{k}+\alpha}^{n+\tau_{k}+\alpha-1}\left[a_{1}(l)-b_{1}(l) x_{1}(l)\right. \\
&\left.+c_{12}(l) \frac{x_{2}(l)}{d_{12}+x_{2}(l)}\right],
\end{aligned}
$$




$$
\begin{aligned}
x_{2}\left(n+\tau_{k}+\alpha\right) & \\
=x_{2}\left(\tau_{k}+\alpha\right) \exp \sum_{l=\tau_{k}+\alpha}^{n+\tau_{k}+\alpha-1} & {\left[a_{2}(l)-b_{2}(l) x_{2}(l)\right.} \\
& \left.+c_{21}(l) \frac{x_{1}(l)}{d_{21}+x_{1}(l)}\right] .
\end{aligned}
$$

Thus, it derives that

$$
\begin{aligned}
x_{1}(n+ & \left.\tau_{k}+\alpha\right) \\
= & x_{1}\left(\tau_{k}+\alpha\right) \\
& \quad \times \exp \sum_{l=\alpha}^{n+\alpha-1}\left[a_{1}\left(l+\tau_{k}\right)-b_{1}\left(l+\tau_{k}\right) x_{1}\left(l+\tau_{k}\right)\right. \\
& \left.+c_{12}\left(l+\tau_{k}\right) \frac{x_{2}\left(l+\tau_{k}\right)}{d_{12}+x_{2}\left(l+\tau_{k}\right)}\right], \\
x_{2}(n+ & \left.\tau_{k}+\alpha\right) \quad x_{2}\left(\tau_{k}+\alpha\right) \quad \\
& \times \exp \sum_{l=\alpha}^{n+\alpha-1}\left[a_{2}\left(l+\tau_{k}\right)-b_{2}\left(l+\tau_{k}\right) x_{2}\left(l+\tau_{k}\right)\right. \\
& \left.\quad+c_{21}\left(l+\tau_{k}\right) \frac{x_{1}\left(l+\tau_{k}\right)}{d_{21}+x_{1}\left(l+\tau_{k}\right)}\right] .
\end{aligned}
$$

Let $k \rightarrow+\infty$; we have

$$
\begin{array}{r}
x_{1}^{*}(n+\alpha) \\
=x_{1}^{*}(\alpha) \exp \sum_{l=\alpha}^{n+\alpha-1}\left[a_{1}(l)-b_{1}(l) x_{1}^{*}(l)\right. \\
\left.+c_{12}(l) \frac{x_{2}^{*}(l)}{d_{12}+x_{2}^{*}(l)}\right], \\
x_{2}^{*}(n+\alpha) \\
=x_{2}^{*}(\alpha) \exp \sum_{l=\alpha}^{n+\alpha-1}\left[a_{2}(l)-b_{2}(l) x_{2}^{*}(l)\right. \\
\left.+c_{21}(l) \frac{x_{1}^{*}(l)}{d_{21}+x_{1}^{*}(l)}\right] .
\end{array}
$$

Since $\alpha$ is arbitrary, we know that $\left(x_{1}^{*}(n), x_{2}^{*}(n)\right)$ is a solution of system (54) on $Z^{+}$, and

$$
\begin{array}{r}
0<m_{1}-\varepsilon \leq x_{1}^{*}(n) \leq M_{1}+\varepsilon, \\
0<m_{2}-\varepsilon \leq x_{2}^{*}(n) \leq M_{2}+\varepsilon, \\
\forall n \in Z^{+} .
\end{array}
$$

Notice that $\varepsilon$ is an arbitrarily small positive constant; it follows that

$$
\begin{array}{r}
0<m_{1} \leq x_{1}^{*}(n) \leq M_{1}, \\
0<m_{2} \leq x_{2}^{*}(n) \leq M_{2}, \\
\forall n \in Z^{+} .
\end{array}
$$

Thus, $\Omega \neq \Phi$. This completes the proof.

Theorem 16. Assume that (H1) holds; furthermore, $0<\beta<1$, where $\beta=\min \left\{\beta_{1}, \beta_{2}\right\}$, and

$$
\begin{gathered}
\beta_{i}=b_{i}^{l} m_{i}-\left(b_{i}^{u}\right)^{2} M_{i}^{2}-\frac{d_{i j} c_{i j}^{u} M_{j}\left(1+b_{i}^{u} M_{i}\right)}{\left(d_{i j}+m_{j}\right)^{2}} \\
-\frac{d_{j i} c_{j i}^{u} M_{i}\left(1+b_{j}^{u} M_{j}+d_{j i} c_{j i}^{u} M_{i}\right)}{\left(d_{j i}+m_{i}\right)^{2}}, \\
i, j=1,2, \quad i \neq j .
\end{gathered}
$$

Then, there exists a unique uniformly asymptotically stable almost periodic solution of system (54) which is bounded by $\Omega$ for all $n \in Z^{+}$.

Proof. Denote $p_{1}(n)=\ln x_{1}(n), p_{2}(n)=\ln x_{2}(n)$. It follows from system (54) that

$$
\begin{aligned}
p_{1}(n+1)= & p_{1}(n)+a_{1}(n)-b_{1}(n) e^{p_{1}(n)} \\
& +c_{12}(n) \frac{e^{p_{2}(n)}}{d_{12}+e^{p_{2}(n)}}, \\
p_{2}(n+1)= & p_{2}(n)+a_{2}(n)-b_{2}(n) e^{p_{2}(n)} \\
& +c_{21}(n) \frac{e^{p_{1}(n)}}{d_{21}+e^{p_{1}(n)}} .
\end{aligned}
$$

According to Proposition 15, we can see that the system (64) has a bounded solution $\left(p_{1}(n), p_{2}(n)\right)$ satisfying

$$
\ln m_{i} \leq \ln p_{i}(n) \leq \ln M_{i}, \quad i=1,2, \forall n \in Z^{+} .
$$

Thus, $\left|p_{i}(n)\right| \leq \overline{M_{i}}$, where $\overline{M_{i}}=\max \left\{\left|\ln m_{i}\right|,\left|\ln M_{i}\right|\right\}, i=$ 1,2 .

Define the norm $\left\|\left(p_{1}(n), p_{2}(n)\right)\right\|=\left|p_{1}(n)\right|+\left|p_{2}(n)\right|$, where $\left(p_{1}(n), p_{2}(n)\right) \in R^{2}$. Consider the product system of system (64) as follows:

$$
\begin{aligned}
p_{1}(n+1)= & p_{1}(n)+a_{1}(n)-b_{1}(n) e^{p_{1}(n)} \\
& +c_{12}(n) \frac{e^{p_{2}(n)}}{d_{12}+e^{p_{2}(n)}}, \\
p_{2}(n+1)= & p_{2}(n)+a_{2}(n)-b_{2}(n) e^{p_{2}(n)} \\
& +c_{21}(n) \frac{e^{p_{1}(n)}}{d_{21}+e^{p_{1}(n)}},
\end{aligned}
$$




$$
\begin{aligned}
q_{1}(n+1)= & q_{1}(n)+a_{1}(n)-b_{1}(n) e^{q_{1}(n)} \\
& +c_{12}(n) \frac{e^{q_{2}(n)}}{d_{12}+e^{q_{2}(n)}}, \\
q_{2}(n+1)= & q_{2}(n)+a_{2}(n)-b_{2}(n) e^{q_{2}(n)} \\
& +c_{21}(n) \frac{e^{q_{1}(n)}}{d_{21}+e^{q_{1}(n)}} .
\end{aligned}
$$

We assume that $Q=\left(p_{1}(n), p_{2}(n)\right), W=\left(q_{1}(n), q_{2}(n)\right)$ are any two solutions of system (66) defined on $S^{*}$; then, $\|Q\| \leq B$, $\|W\| \leq B$, where $B=\overline{M_{1}}+\overline{M_{2}}$, and $S^{*}=\left\{\left(p_{1}(n), p_{2}(n)\right) \mid\right.$ $\left.\ln m_{i} \leq p_{i}(n) \leq \ln M_{i}, i=1,2, n \in Z^{+}\right\}$.

Let us construct a Lyapunov function defined on $Z^{+} \times S^{*} \times$ $S^{*}$ as follows:

$$
V(n, Q, W)=\left(p_{1}(n)-q_{1}(n)\right)^{2}+\left(p_{2}(n)-q_{2}(n)\right)^{2} .
$$

It is obvious that the norm $\|Q-W\|=\left|p_{1}(n)-q_{1}(n)\right|+\mid p_{2}(n)-$ $q_{2}(n) \mid$ is equivalent to $\|Q-W\|_{*}=\left[\left(p_{1}(n)-q_{1}(n)\right)^{2}+\left(p_{2}(n)-\right.\right.$ $\left.\left.q_{2}(n)\right)^{2}\right]^{1 / 2}$; that is, there are two constants $c_{1}>0, c_{2}>0$, such that

$$
c_{1}\|Q-W\| \leq\|Q-W\|_{*} \leq c_{2}\|Q-W\| ;
$$

then,

$$
\left(c_{1}\|Q-W\|\right)^{2} \leq V(n, Q, W) \leq\left(c_{2}\|Q-W\|\right)^{2} .
$$

Let $\psi, \varphi \in C\left(R^{+}, R^{+}\right), \psi(x)=c_{1}^{2} x^{2}, \varphi=c_{2}^{2} x^{2}$; then, condition (i) of Lemma 10 is satisfied. have

Moreover, for any $(n, Q, W),(n, \bar{Q}, \bar{W}) \in Z^{+} \times S^{*} \times S^{*}$, we

$$
\begin{aligned}
&|V(n, Q, W)-V(n, \bar{Q}, \bar{W})| \\
&=\mid\left(p_{1}(n)-q_{1}(n)\right)^{2}+\left(p_{2}(n)-q_{2}(n)\right)^{2} \\
& \quad-\left(\overline{p_{1}}(n)-\overline{q_{1}}(n)\right)^{2}-\left(\overline{p_{2}}(n)-\overline{q_{2}}(n)\right)^{2} \mid \\
& \leq \quad\left[\left|p_{1}(n)\right|+\left|q_{1}(n)\right|+\left|\overline{p_{1}}(n)\right|+\left|\overline{q_{1}}(n)\right|\right] \\
& \times \quad\left[\left|p_{1}(n)-\overline{p_{1}}(n)\right|+\left|q_{1}(n)-\overline{q_{1}}(n)\right|\right] \\
&+\quad\left[\left|p_{2}(n)\right|+\left|q_{2}(n)\right|+\left|\overline{p_{2}}(n)\right|+\left|\overline{q_{2}}(n)\right|\right] \\
& \times {\left[\left|p_{2}(n)-\overline{p_{2}}(n)\right|+\left|q_{2}(n)-\overline{q_{2}}(n)\right|\right] } \\
& \leq L\left[\left|p_{1}(n)-\overline{p_{1}}(n)\right|+\left|p_{2}(n)-\overline{p_{2}}(n)\right|\right. \\
&\left.\quad+\left|q_{1}(n)-\overline{q_{1}}(n)\right|+\left|q_{2}(n)-\overline{q_{2}}(n)\right|\right] \\
&= L(\|Q-\bar{Q}||+|| W-\bar{W}\|),
\end{aligned}
$$

where $\bar{Q}\left(\overline{p_{1}}(n), \overline{p_{2}}(n)\right), \bar{W}\left(\overline{q_{1}}(n), \overline{q_{2}}(n)\right)$, and $L=4 \max \left\{\overline{M_{1}}\right.$, $\left.\overline{M_{2}}\right\}$. Thus, condition (ii) of Lemma 10 is satisfied.
Finally, calculating the $\Delta V(n)$ of $V(n)$ along the solutions of system (64), we have

$$
\begin{aligned}
& \Delta V(n)=V(n+1)-V(n) \\
& =\left[\left(p_{1}(n+1)-q_{1}(n+1)\right)^{2}\right. \\
& \left.+\left(p_{2}(n+1)-q_{2}(n+1)\right)^{2}\right] \\
& -\left[\left(p_{1}(n)-q_{1}(n)\right)^{2}+\left(p_{2}(n)-q_{2}(n)\right)^{2}\right] \\
& =\sum_{i=1, j \neq i}^{2}\left[-2 b_{i}(n)\left(p_{i}(n)-q_{i}(n)\right)\left(e^{p_{i}(n)}-e^{q_{i}(n)}\right)\right. \\
& +b_{i}^{2}(n)\left(e^{p_{i}(n)}-e^{q_{i}(n)}\right)^{2}+2 c_{i j}(n) \\
& \times\left[p_{i}(n)-q_{i}(n)\right. \\
& \left.-b_{i}(n)\left(e^{p_{i}(n)}-e^{q_{i}(n)}\right)\right] \\
& \times \frac{d_{i j}\left(e^{p_{j}(n)}-e^{q_{j}(n)}\right)}{\left(d_{i j}+e^{p_{j}(n)}\right)\left(d_{i j}+e^{q_{j}(n)}\right)} \\
& \left.+c_{i j}^{2}(n) \frac{d_{i j}^{2}\left(e^{p_{j}(n)}-e^{q_{j}(n)}\right)^{2}}{\left(d_{i j}+e^{p_{j}(n)}\right)^{2}\left(d_{i j}+e^{q_{j}(n)}\right)^{2}}\right] .
\end{aligned}
$$

By the mean value theorem, it derives that

$$
e^{p_{i}(n)}-e^{q_{i}(n)}=\xi_{i}(n)\left(p_{i}(n)-q_{i}(n)\right), \quad i=1,2,
$$

where $\xi_{i}(n)$ lies between $e^{p_{i}(n)}$ and $e^{q_{i}(n)}$. Then,

$$
\left.\begin{array}{rl}
\Delta V(n)=\sum_{i=1, j \neq i}^{2}\left[\begin{array}{c}
- \\
-
\end{array} b_{i}(n) \xi_{i}(n)\left(p_{i}(n)-q_{i}(n)\right)^{2}\right. \\
+b_{i}^{2}(n) \xi_{i}^{2}(n)\left(p_{i}(n)-q_{i}(n)\right)^{2} \\
+\left(\left(2 d_{i j} c_{i j}(n) \xi_{j}(n)\right.\right. \\
\times\left(p_{i}(n)-q_{i}(n)\right) \\
\times\left(p_{j}(n)-q_{j}(n)\right) \\
\left.\times\left[1-b_{i}(n) \xi_{i}(n)\right]\right) \\
\times\left(\left(d_{i j}+e^{p_{j}(n)}\right)\right. \\
\left.\left.\times\left(d_{i j}+e^{q_{j}(n)}\right)\right)^{-1}\right) \\
+\frac{d_{i j}^{2} c_{i j}^{2}(n) \xi_{j}^{2}(n)\left(p_{j}(n)-q_{j}(n)\right)^{2}}{\left(d_{i j}+e^{p_{j}(n)}\right)^{2}\left(d_{i j}+e^{q_{j}(n)}\right)^{2}}
\end{array}\right]
$$




$$
\begin{aligned}
& \leq \sum_{i=1, j \neq i}^{2}\left[-2 b_{i}(n) \xi_{i}(n)\left(p_{i}(n)-q_{i}(n)\right)^{2}\right. \\
& +b_{i}^{2}(n) \xi_{i}^{2}(n)\left(p_{i}(n)-q_{i}(n)\right)^{2} \\
& +\frac{d_{i j} c_{i j}(n) \xi_{j}(n)\left[1+b_{i}(n) \xi_{i}(n)\right]}{\left(d_{i j}+m_{j}\right)^{2}} \\
& \times\left[\left(p_{i}(n)-q_{i}(n)\right)^{2}\right. \\
& \left.+\left(p_{j}(n)-q_{j}(n)\right)^{2}\right] \\
& \left.+\frac{d_{i j}^{2} c_{i j}^{2}(n) \xi_{j}^{2}(n)}{\left(d_{i j}+m_{j}\right)^{2}}\left(p_{j}(n)-q_{j}(n)\right)^{2}\right] \\
& \leq \sum_{i=1, j \neq i}^{2}\left[\left[-2 b_{i}(n) \xi_{i}(n)+b_{i}^{2}(n) \xi_{i}^{2}(n)\right.\right. \\
& \left.+\frac{d_{i j} c_{i j}(n) \xi_{j}(n)\left[1+b_{i}(n) \xi_{i}(n)\right]}{\left(d_{i j}+m_{j}\right)^{2}}\right] \\
& \times\left(p_{i}(n)-q_{i}(n)\right)^{2} \\
& +\left(\left(d_{i j} c_{i j}(n) \xi_{j}(n)\right.\right. \\
& \times\left[1+b_{i}(n) \xi_{i}(n)\right. \\
& \left.\left.+d_{i j} c_{i j}(n) \xi_{j}(n)\right]\right) \\
& \left.\times\left(d_{i j}+m_{j}\right)^{-2}\right) \\
& \left.\times\left(p_{j}(n)-q_{j}(n)\right)^{2}\right] \\
& \leq \sum_{i=1, j \neq i}^{2}\left[\left[-2 b_{i}^{l} m_{i}+\left(b_{i}^{u}\right)^{2} M_{i}^{2}\right.\right. \\
& \left.+\frac{d_{i j} c_{i j}^{u} M_{j}\left(1+b_{i}^{u} M_{i}\right)}{\left(d_{i j}+m_{j}\right)^{2}}\right] \\
& \times\left(p_{i}(n)-q_{i}(n)\right)^{2} \\
& +\frac{d_{i j} c_{i j}^{u} M_{j}\left(1+b_{i}^{u} M_{i}+d_{i j} c_{i j}^{u} M_{j}\right)}{\left(d_{i j}+m_{j}\right)^{2}} \\
& \left.\times\left(p_{j}(n)-q_{j}(n)\right)^{2}\right] \\
& \leq-\sum_{i=1}^{2} \beta_{i}\left(p_{i}(n)-q_{i}(n)\right)^{2} \leq-\beta V(n),
\end{aligned}
$$

where $\beta=\min \left\{\beta_{1}, \beta_{2}\right\}$. By the conditions of Theorem 16, we have $0<\beta<1$, and hence condition (iii) of Lemma 10 is satisfied. So, it follows from Lemma 10 that there exists a unique uniformly asymptotically stable almost periodic solution $\left(p_{1}^{*}(n), p_{2}^{*}(n)\right)$ of system (64) which is bounded by $S^{*}$ for all $n \in Z^{+}$; that is, there exists a unique uniformly asymptotically stable almost periodic solution $\left(x_{1}^{*}(n), x_{2}^{*}(n)\right)$ of system (54) which is bounded by $\Omega$ for all $n \in Z^{+}$. This completed the proof.

\section{Numerical Simulations}

In this section, we give the following example to check the feasibility of our result.

Example 1. Consider the discrete multispecies Lotka-Volterra mutualism system:

$$
\begin{aligned}
x_{1}(k+1) & \\
=x_{1}(k) \exp \{1 & 1.2-0.02 \sin (\sqrt{2} k) \\
& -(1.05+0.01 \sin (\sqrt{3} k)) x_{1}(k) \\
& +(0.025+0.002 \cos (\sqrt{5} k)) \\
& \times \frac{x_{2}(k)}{1.1+x_{2}(k)} \\
& +(0.02+0.001 \cos (\sqrt{2} k)) \\
& \left.\times \frac{x_{3}(k)}{1.2+x_{3}(k)}\right\}
\end{aligned}
$$

$x_{2}(k+1)$

$$
=x_{2}(k) \exp \{1.1-0.025 \cos (\sqrt{3} k)
$$$$
-(1.08+0.015 \sin (\sqrt{2} k)) x_{2}(k)
$$$$
+(0.02+0.003 \sin (\sqrt{2} k))
$$$$
\times \frac{x_{1}(k)}{1.4+x_{1}(k)}
$$$$
+(0.025+0.002 \cos (\sqrt{5} k))
$$$$
\left.\times \frac{x_{3}(k)}{1.6+x_{3}(k)}\right\} \text {, }
$$

$x_{3}(k+1)$

$$
\begin{aligned}
=x_{3}(k) \exp \{ & 1.15-0.03 \cos (\sqrt{5} k) \\
& -(1.1+0.02 \cos (\sqrt{2} n)) x_{1}(k)
\end{aligned}
$$




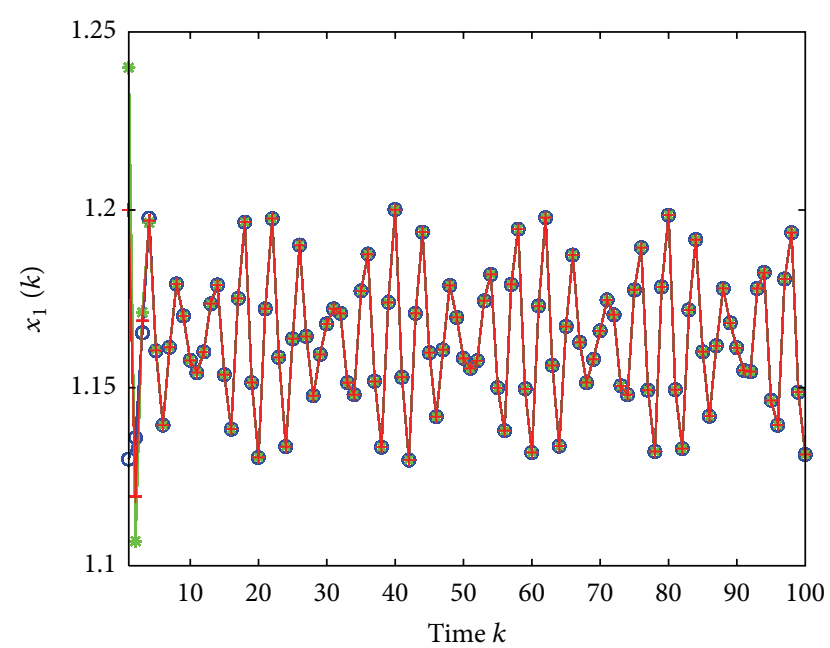

Figure 1: Dynamic behavior of the first component $x_{1}(k)$ of the solution $\left(x_{1}(k), x_{2}(k), x_{3}(k)\right)$ to system (74) with the initial conditions $(1.13,1.17,1.2),(1.24,0.96,0.95)$, and $(1.2,1.08,1.14)$ for $k \in[1,100]$, respectively.

$$
\begin{aligned}
& +(0.03+0.0025 \sin (\sqrt{2} k)) \\
& \times \frac{x_{1}(k)}{2.1+x_{1}(k)} \\
& +(0.028+0.0015 \sin (\sqrt{3} k)) \\
& \left.\times \frac{x_{2}(k)}{1.8+x_{2}(k)}\right\} .
\end{aligned}
$$

A computation shows that

$$
\begin{array}{ll}
m_{1} \approx 0.9538, & M_{1} \approx 1.257, \\
m_{2} \approx 0.9817, & M_{2} \approx 1.1185, \\
m_{3} \approx 0.818, & M_{3} \approx 1.1794,
\end{array}
$$

and, moreover, we have that

$$
\rho_{1} \approx 0.3857, \quad \rho_{2} \approx 0.2856, \quad \rho_{3} \approx 0.3947,
$$

and that $\max \left\{\rho_{1}, \rho_{2}, \rho_{3}\right\} \approx 0.3947<1$. It is easy to see that condition (H2) is satisfied. Hence, there exists a unique globally attractive almost periodic solution of system (74). Our numerical simulations support our results (see Figures 1,2 , and 3 ).

\section{Conflict of Interests}

The authors declare that there is no conflict of interests regarding the publication of this paper, and there is no financial conflict of interests between the authors and the commercial identity.

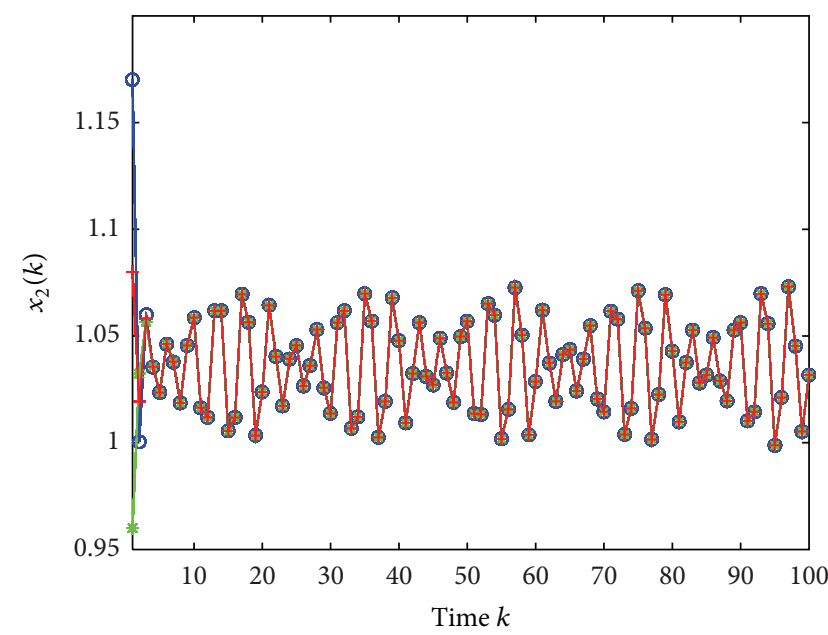

FIGURE 2: Dynamic behavior of the second component $x_{2}(k)$ of the solution $\left(x_{1}(k), x_{2}(k), x_{3}(k)\right)$ to system (74) with the initial conditions $(1.13,1.17,1.2),(1.24,0.96,0.95)$, and $(1.2,1.08,1.14)$ for $k \in[1,100]$, respectively.

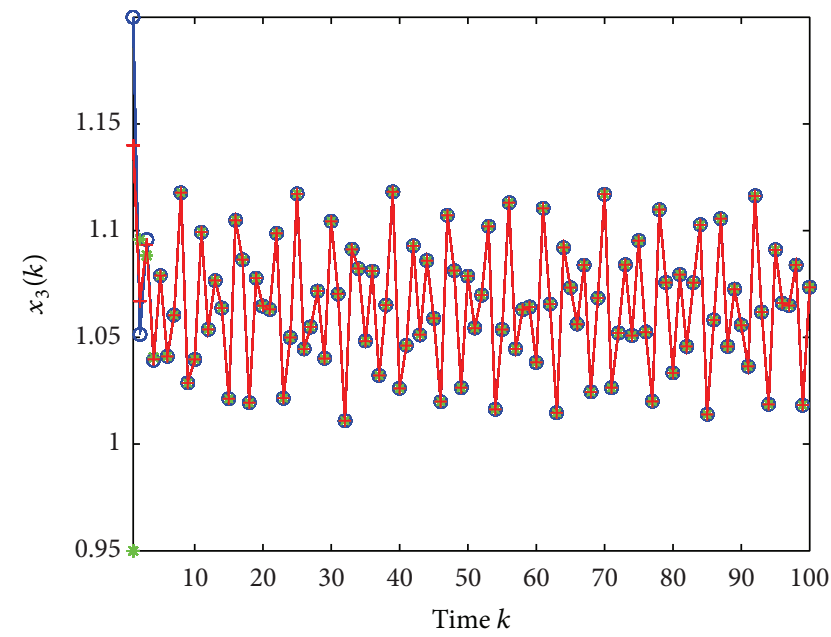

FIgURE 3: Dynamic behavior of the third component $x_{3}(k)$ of the solution $\left(x_{1}(k), x_{2}(k), x_{3}(k)\right)$ to system (74) with the initial conditions $(1.13,1.17,1.2),(1.24,0.96,0.95)$, and $(1.2,1.08,1.14)$ for $k \in[1,100]$, respectively.

\section{Acknowledgments}

This work is supported by the National Natural Science Foundation of China (no. 61132008) and the Scientific Research Program Funded by Shaanxi Provincial Education Department of China (no. 2013JK1098).

\section{References}

[1] W. Wendi and L. Zhengyi, "Global stability of discrete models of Lotka-Volterra type," Nonlinear Analysis: Theory, Methods and Applications, vol. 35, no. 8, pp. 1019-1030, 1999.

[2] Y. Li, "Positive periodic solutions of a discrete mutualism model with time delays," International Journal of Mathematics and Mathematical Sciences, vol. 2005, no. 4, pp. 499-506, 2005. 
[3] F. Chen, "Permanence and global attractivity of a discrete multispecies Lotka-Volterra competition predator-prey systems," Applied Mathematics and Computation, vol. 182, no. 1, pp. 3-12, 2006.

[4] Y. Muroya, "Persistence and global stability in discrete models of Lotka-Volterra type," Journal of Mathematical Analysis and Applications, vol. 330, no. 1, pp. 24-33, 2007.

[5] F. Chen, "Permanence for the discrete mutualism model with time delays," Mathematical and Computer Modelling, vol. 47, no. 3-4, pp. 431-435, 2008.

[6] K. Mukdasai, "Robust exponential stability for LPD discretetime system with interval time-varying delay," Journal of Applied Mathematics, vol. 2012, Article ID 237430, 13 pages, 2012.

[7] Q. Wang and Z. Liu, "Uniformly asymptotic stability of positive almost periodic solutions for a discrete competitive system," Journal of Applied Mathematics, vol. 2013, Article ID 182158, 9 pages, 2013.

[8] H. Zhang, Y. Li, and B. Jing, "Global attractivity and almost periodic solution of a discrete mutualism model with delays," Mathematical Methods in the Applied Sciences, 2013.

[9] Y. Li and G. Xu, "Positive periodic solutions for an integrodifferential model of mutualism," Applied Mathematics Letters, vol. 14, no. 5, pp. 525-530, 2001.

[10] Y. Xia, J. Cao, and S. S. Cheng, "Periodic solutions for a Lotka-Volterra mutualism system with several delays," Applied Mathematical Modelling, vol. 31, no. 9, pp. 1960-1969, 2007.

[11] F. Chen, "Permanence for the discrete mutualism model with time delays," Mathematical and Computer Modelling, vol. 47, no. 3-4, pp. 431-435, 2008.

[12] Y. Li and H. Zhang, "Existence of periodic solutions for a periodic mutualism model on time scales," Journal of Mathematical Analysis and Applications, vol. 343, no. 2, pp. 818-825, 2008.

[13] H. Zhang, Y. Li, B. Jing, and W. Zhao, "Global stability of almost periodic solution of multispecies mutualism system with time delays and impulsive effects," Applied Mathematics and Computation, vol. 232, pp. 1138-1150, 2014.

[14] Z. Li and F. Chen, "Almost periodic solutions of a discrete almost periodic logistic equation," Mathematical and Computer Modelling, vol. 50, no. 1-2, pp. 254-259, 2009.

[15] C. Niu and X. Chen, "Almost periodic sequence solutions of a discrete Lotka-Volterra competitive system with feedback control," Nonlinear Analysis: Real World Applications, vol. 10, no. 5, pp. 3152-3161, 2009.

[16] Z. Li, F. Chen, and M. He, "Almost periodic solutions of a discrete Lotka-Volterra competition system with delays," Nonlinear Analysis: Real World Applications, vol. 12, no. 4, pp. 2344-2355, 2011.

[17] Y. Li and T. Zhang, "Permanence and almost periodic sequence solution for a discrete delay logistic equation with feedback control," Nonlinear Analysis: Real World Applications, vol. 12, no. 3, pp. 1850-1864, 2011.

[18] Y. Li, T. Zhang, and Y. Ye, "On the existence and stability of a unique almost periodic sequence solution in discrete predatorprey models with time delays," Applied Mathematical Modelling, vol. 35, no. 11, pp. 5448-5459, 2011.

[19] T. Zhang, Y. Li, and Y. Ye, "Persistence and almost periodic solutions for a discrete fishing model with feedback control," Communications in Nonlinear Science and Numerical Simulation, vol. 16, no. 3, pp. 1564-1573, 2011.

[20] Y. Wang, "Periodic and almost periodic solutions of a nonlinear single species discrete model with feedback control," Applied
Mathematics and Computation, vol. 219, no. 10, pp. 5480-5486, 2013.

[21] T. Zhang and X. Gan, "Almost periodic solutions for a discrete fishing model with feedback control and time delays," Communications in Nonlinear Science and Numerical Simulation, vol. 19, no. 1, pp. 150-163, 2014.

[22] A. M. Fink and G. Seifert, "Liapunov functions and almost periodic solutions for almost periodic systems," Journal of Differential Equations, vol. 5, pp. 307-313, 1969.

[23] R. Yuan, "The existence of almost periodic solutions of retarded differential equations with piecewise constant argument," Nonlinear Analysis, Theory, Methods and Applications, vol. 48, no. 7, pp. 1013-1032, 2002.

[24] L. Wu, F. Chen, and Z. Li, "Permanence and global attractivity of a discrete Schoener's competition model with delays," Mathematical and Computer Modelling, vol. 49, no. 7-8, pp. 1607-1617, 2009.

[25] A. M. Samoilenko and N. A. Perestyuk, Impulsive Differential Equations, World Scientific Series on Nonlinear Science, World Scientific, Singapore, 1995.

[26] S. N. Zhang and G. Zheng, "Almost periodic solutions of delay difference systems," Applied Mathematics and Computation, vol. 131, no. 2-3, pp. 497-516, 2002.

[27] Z. Zhou and X. Zou, "Stable periodic solutions in a discrete periodic logistic equation," Applied Mathematics Letters, vol. 16, no. 2, pp. 165-171, 2003.

[28] S. Zhang, "Existence of almost periodic solutions for difference systems," Annals of Differential Equations, vol. 16, no. 2, pp. 184206, 2000 . 


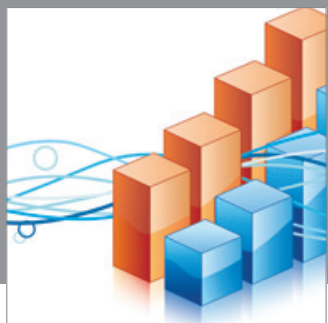

Advances in

Operations Research

mansans

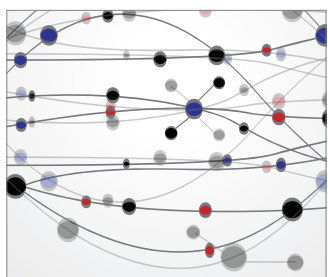

The Scientific World Journal
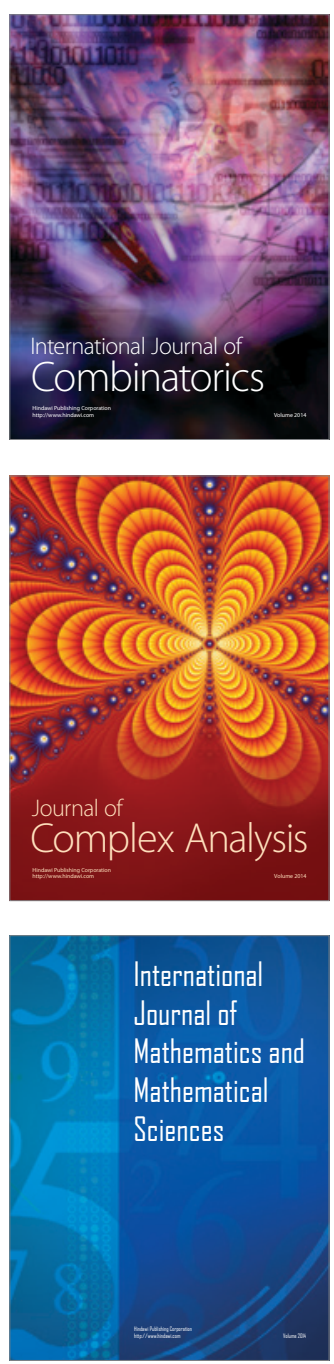
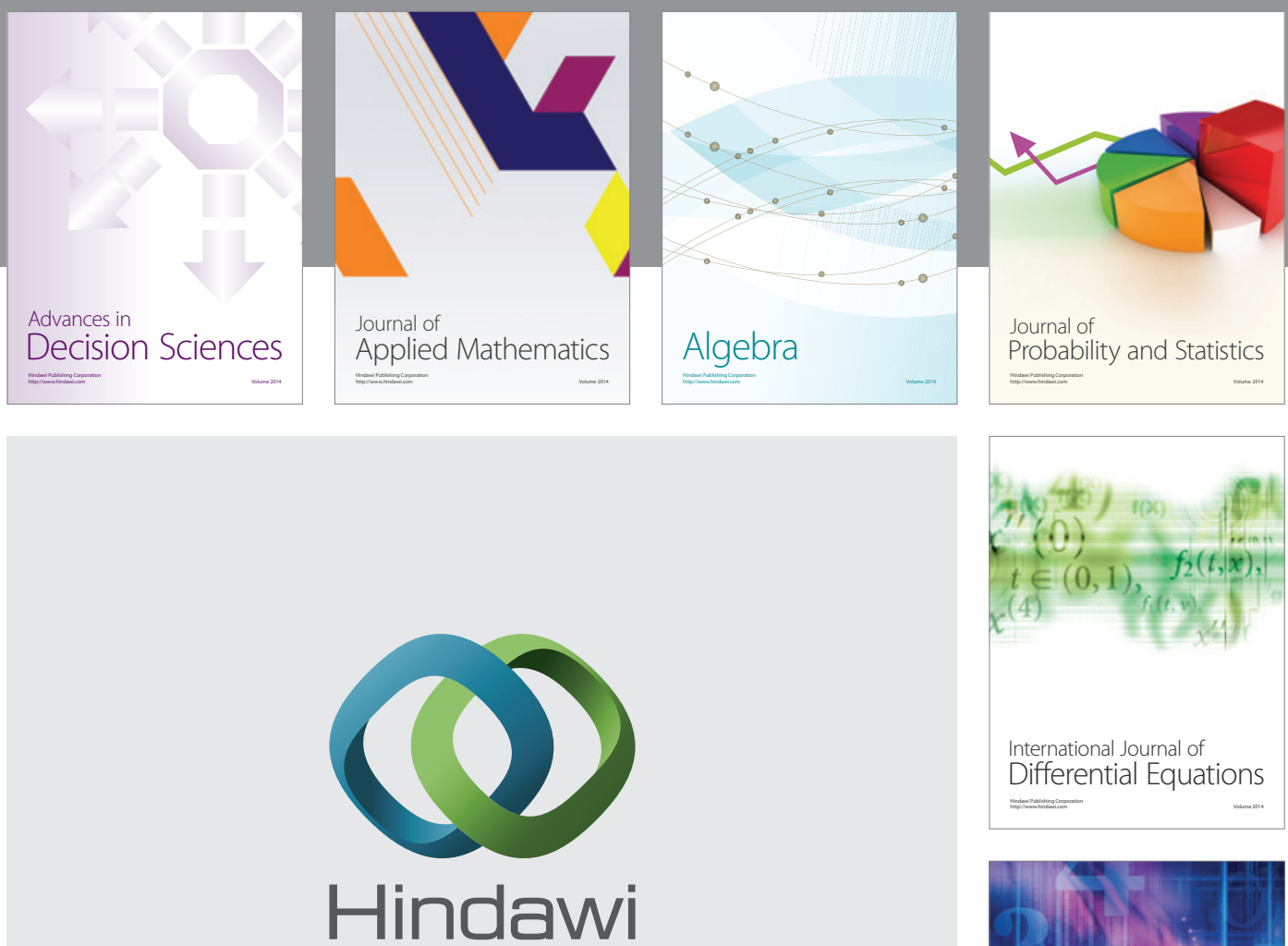

Submit your manuscripts at http://www.hindawi.com
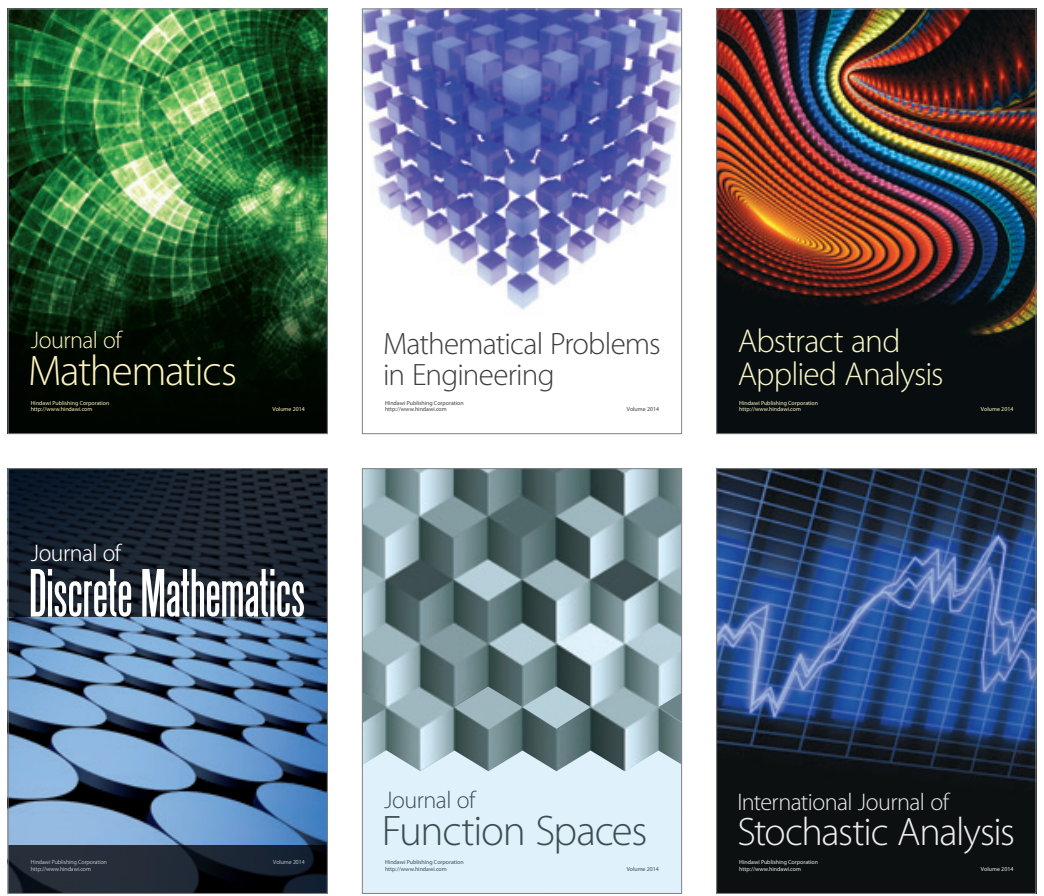

Journal of

Function Spaces

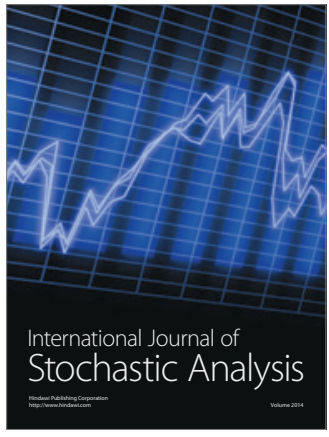

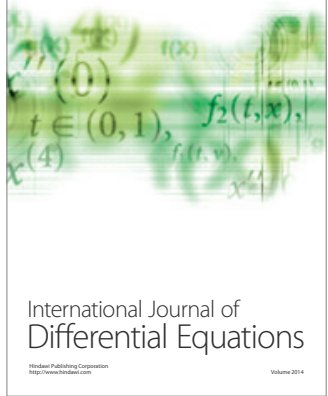
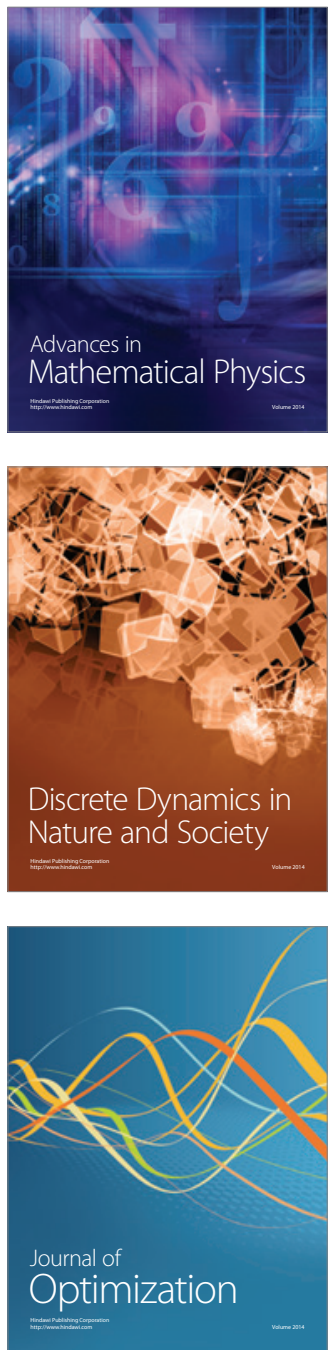